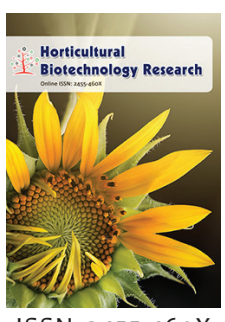

ISSN: $2455-460 \mathrm{X}$

Received: April 08, 2019 Accepted: May 03, 2019 Published: May 05, 2019

*Corresponding Author: May Sandar Kyaing, Email: maysandar1207@ gmail.com

\section{Regional polymorphism assessment of Myanmar 'Sein Ta Lone' mango (Mangifera indica Linn) from Sintgaing Township based on microsatellite markers}

\author{
Seinn Sandar May Phyo, Nwe Nwe Soe Hlaing, May Sandar Kyaing*, \\ Moe Moe Myint, April Nwet Yee Soe, Honey Thet Paing Htway, \\ Khin Pyone Yi
}

Molecular Genetics Laboratory, Biotechnology Research Department, Kyaukse, Mandalay Region, Myanmar

\begin{abstract}
This study was conducted to explore the genetic diversity and relationship of Sein Ta Lone mango cultivars among 20 commercial orchards in Sintgaing Township, Mandalay region. Nine microsatellite (SSR) markers were used to detect genetic polymorphism in a range from ( 3 to 6 ) alleles with (4.33) alleles per marker in average. Six out of nine microsatellite markers gave the PIC values of greater than (0.5). Among them, SSR36 held the highest PIC values of (0.691) while MiSHRS39 and MN85 possessed the least PIC values of (0.368) and (0.387) respectively. The genetic diversity was expressed as unbiased expected heterozygosity (UHe) value with an average of $(0.561)$. The genetic relationship was revealed by (UPGMA) dendrogram in a range of (0.69 to 1.00). Based on UPGMA cluster analysis, three main clusters were classified among three different locations. This study was intended to help cultivar characterization and conservation for proper germplasm management with the estimation of genetic variation and relationship in the existing population of Sein Ta Lone mangoes in Sintgaing Township by microsatellite markers.
\end{abstract}

KEYWORDS: Polymorphism assessment, Sein Ta Lone, microsatellites (SSR) markers, Sintgaing Township

\section{INTRODUCTION}

Mango (Mangifera indica L.) plays an important horticultural fruits in Myanmar. Popenoe W (1920) [18] stated that it is originated as an allopolyploid from eastern India, Assam and Myanmar. Indo-Burma region was suggested as the centre of origin of mango based on the observed level of genetic diversity as described by Vavilov NI (1926) [26]. Mango has rich germplasm diversity and there are about 1600 varieties in the world [16]. Hirano et al (2010) [6] reported that Myanmar has been expected to have broad national - level of genetic diversity through the long history of mango cultivation. About 300 cultivars and 20 kinds of mango species are found in Myanmar described by Soe TT (2006) [24]. Mango can grow well throughout the country under the various climatic conditions as it is a native kind of fruit in Myanmar. Myat $\mathrm{K}$ (2012)[13] also mentioned that because of the region's prevalently hot climate and the exportable quality, mangoes from Mandalay region are considered to be the best quality of mangoes.

Sein Ta Lone is known as One Diamond in the local language. As its name implies, it is the most popular and superior horticultural mango in Myanmar because of its nutritional quality, taste and consumer's preferences such as delightful aroma, sweetness, juiciness and non-fibrous nature. Moreover, Sein Ta Lone becomes famous and attractive to global economy due to its excellent quality. Along with the trade expansion of fresh mangoes, there has been a problem for several constraints including intravarietal variability in which locality dependent variations were found in the fruit size, shape and quality. As variations in morphological traits and physio-chemical factors among intra-cultivars of mango were most likely to be due to changes in genetic characters, it is essential to study the genetic variation among intra-cultivars of Sein Ta Lone mango in Myanmar.

Copyright: $\odot$ The authors. This article is open access and licensed under the terms of the Creative Commons Attribution License (http://creativecommons.org/licenses/by/4.o/) which permits unrestricted, use, distribution and reproduction in any medium, or format for any purpose, even commercially provided the work is properly cited. Attribution - You must give appropriate credit, provide a link to the license, and indicate if changes were made. 
Molecular markers have been widely used to overcome the problem caused by environmental parameters. Molecular analysis and fingerprinting of mango cultivars with different types of DNA markers, has been attempted for recent years which included random amplified polymorphic DNA (RAPD) [8,11], inter simple sequence repeats (ISSR) [17] and [22], amplified fragment length polymorphism (AFLP) $[4,9]$ and simple sequence repeats (SSR) $[3,21,23]$. Among them, microsatellites (SSR) markers has become the marker of choice for fingerprinting purposes in most plant species [5] due to their high polymorphism, co dominancy and reproducibility. In addition, SSR has been regarded as the greater importance due to their abundant occurrence throughout the genome of all the eukaryotes because of it variation in repeat lengths [19].

The better understanding of genetic variation and phylogeny in Sein Ta Lone cultivar is crucial as it is the basic knowledge of improving its genetic resources which is beneficial for breeding programs. As Sein Ta Lone is mainly distributed in central (Central Myanmar) and southern (Lower Myanmar) parts of Myanmar, Sintgaing Township in Mandalay region which was considered to be the center of origin for mango cultivation in Myanmar was focused as the study area in this research. The assessment of genetic diversity and relationship of Sein Ta Lone mango was conducted by using PCR-based SSR markers which were found as the best tool to approach this study.

\section{MATERIALS AND METHODS}

\section{Sample Collection}

Sintgaing Township covering three locations of Paleik, Sintgaing and Sunye in Mandalay region shown in Figure 1 were selected

as the targeted sampling sites. Sample collection was conducted throughout 20 commercial orchards by simple random sampling method. The collected 40 accessions of mango used in this study were shown in Table 1.

\section{Genomic DNA Extraction}

Initially, the protocol standardization for genomic DNA extraction from mango leaf was done based on the modified cetyltrimethyl ammonium bromide (CTAB) method [10]. The quality and quantity of the isolated DNA were determined by using nanophotometer (IMPLEN p330,UK).

Table 1: Sample collection for 40 mango accessions from 20 individual's orchards within Sintgaing Township along with their GPS records

\begin{tabular}{llccl}
\hline Accession code & Orchard name & \multicolumn{2}{c}{ GPS record $\left(^{\circ}\right)$} & Collection \\
\cline { 2 - 4 } & & Latitude $(\mathrm{N})$ & Longitude (E) & Sites \\
\hline SKO & U Aye Ko & 21.682219 & 96.216774 & Sunye \\
SW & U Phoo Toe & 21.683437 & 96.217447 & Sunye \\
SR & Sunye & 21.68479 & 96.217959 & Sunye \\
SMg & U Mg Mg & 21.684683 & 96.218039 & Sunye \\
SCh & U Chit Shwe & 21.685816 & 96.218196 & Sunye \\
SKM & UKyawMyoTun & 21.812928 & 96.059257 & Paleik \\
SPi & PiTaukYeik & 21.81341 & 96.059299 & Paleik \\
SWS & WarSo & 21.813916 & 96.059368 & Paleik \\
STZ & ThazinNwe & 21.812877 & 96.059194 & Paleik \\
SKa & U Karka & 21.812558 & 96.059103 & Paleik \\
SPB & PawTawMu & 21.7888 & 96.0918 & Sintgaing \\
SJT & Jodu & 21.7848 & 96.0887 & Sintgaing \\
SST & U Swe Tint & 21.7835 & 96.0885 & Sintgaing \\
STO & U Than Oo & 21.7829 & 96.0872 & Sintgaing \\
SPS & PyaeSone & 21.783 & 96.0872 & Sintgaing \\
SNM & TaungPawThar & 21.7811 & 96.0862 & Sintgaing \\
SMT & MibaMyittar & 21.7792 & 96.0831 & Sintgaing \\
SSi & Ma Sint & 21.7713 & 96.0852 & Sintgaing \\
SNa & NayChiOo & 21.7715 & 96.0853 & Sintgaing \\
SBO & U Bo Than & 21.77 & 96.0858 & Sintgaing \\
\hline
\end{tabular}

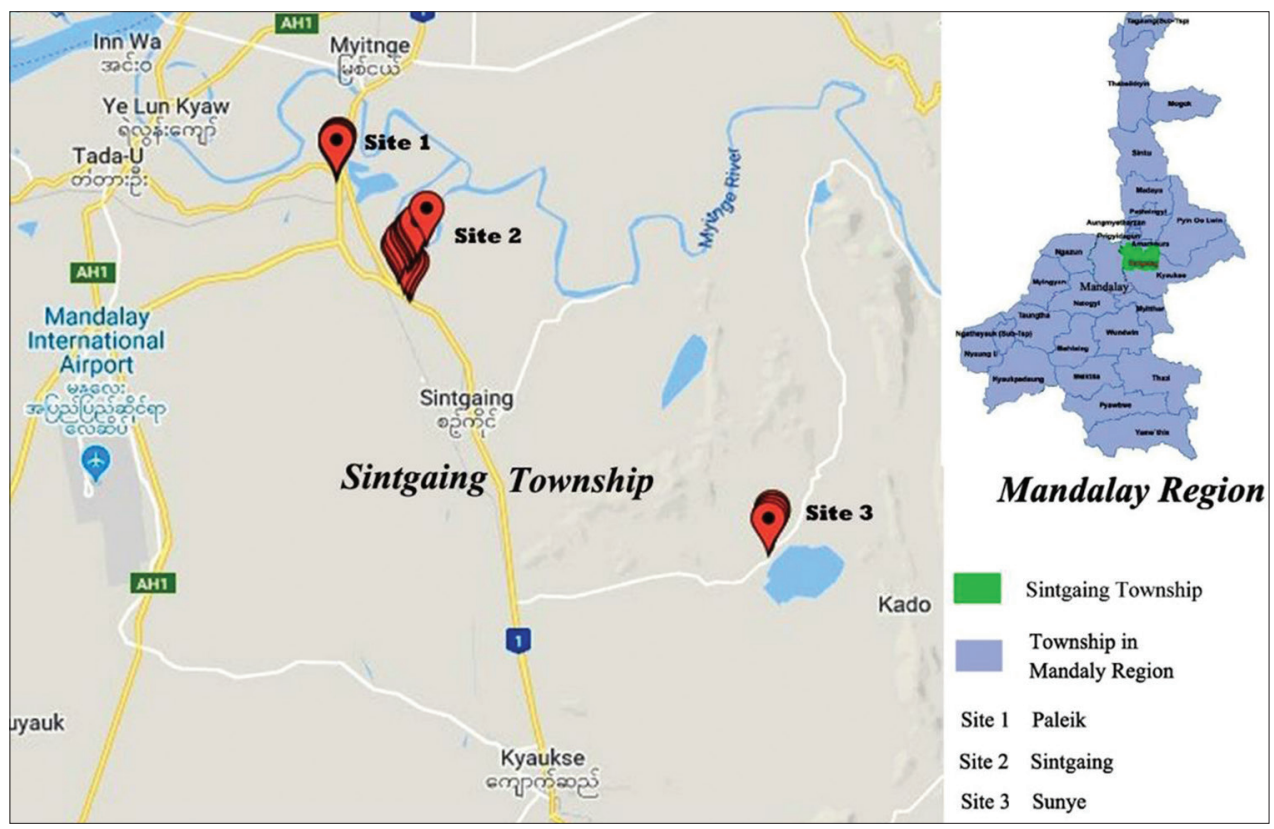

Figure 1: Sample collection sites within Sintgaing Township in Mandalay Region 


\section{SSR Amplification}

Nine SSR markers selected by the previous study were used and listed in Table 2 to reveal genetic polymorphism among the selected mango accessions. SSR analysis was performed in minimum reaction volume. Each PCR reaction contains $10 \mu \mathrm{L}$ in which $1 \mu \mathrm{L}$ of $10 \mathrm{x}$ PCR buffer, $0.8 \mu \mathrm{L}$ of $2.5 \mathrm{mM}$ dNTPs, $0.8 \mu \mathrm{L}$ of $25 \mathrm{mM} \mathrm{MgCl} 2,0.3 \mu \mathrm{L}$ of $5 \mathrm{U}$ Taq polymerase, $1 \mu \mathrm{L}$ of each $10 \mu \mathrm{M}$ forward and reversed primer, $1 \mu \mathrm{L}$ of $25-50 \mathrm{ng} / \mu \mathrm{L}$ of template DNA and $4.1 \mu \mathrm{L}$ of nuclease free water. All PCR reaction-conditions were optimized as initial denaturation at $95^{\circ} \mathrm{C}$ for 4 minutes, followed by 30 cycles of denaturation step at $95^{\circ} \mathrm{C}$ for 30 seconds. The annealing step was set for 30 seconds at their respective annealing temperature (Ta) for each primer in a range of $42-58^{\circ} \mathrm{C}$ which was represented in Table 2 . The extension step was at $72^{\circ} \mathrm{C}$ for 1 minute, then followed by final extension at $72^{\circ} \mathrm{C}$ for 7 minutes. The amplified PCR products were separated on $8 \%$ polyacrylamide gels with 0.5 x TBE buffer at $100 \mathrm{~V}$ for 1 hour. The gels were stained by silver nitrate solution and developer solution $(0.4 \mathrm{M}$ $\mathrm{NaOH} / 4 \%$ Formaldehyde) to visualize the banding patterns. The standard DNA ladder (100 bp) was used to determine the size of the amplified products.

\section{Data Scoring and Statistical Analysis}

The potential for estimating genetic polymorphism was done through counting the clear and reproducible bands visually. The bands produced by these markers were scored by means of the binary codes 0 and 1 at which 1 stands for the presence of an allele, and 0 means the absence of an allele. The number of alleles for each locus and the mean alleles of all loci were recorded for the capacity of the microsatellite markers analyzed. The discriminating power of microsatellite markers used in this study was determined by polymorphic information content (PIC) using the formula $\mathrm{PIC}=1-\Sigma \mathrm{Pi}^{2}$. According to $\mathrm{Ne} \mathrm{M}$ (1974) [14], the unbiased expected heterozygosity values were calculated by $\mathrm{UHe}=[2 \mathrm{~N} /(2 \mathrm{~N}-1)] \times$ He. The dendrogram was constructed based on the Unweighed Pair Group Method and Arithmetic Average (UPGMA) algorithm to deduce genetic relationships among accessions with the help of Numerical Taxonomy and Multivariate Analysis System (NTSYS)-pc, Version 2.02 package [20].

\section{RESULTS AND DISCUSSIONS}

\section{Microsatellite Allele Polymorphism}

A total of 9 microsatellite markers shown in Table 2 were utilized to characterize and assess polymorphism of 40 accessions of Sein Ta Lone mango. The data with respect to polymorphic characteristics detected by these 9 microsatellite markers were described in Table 3. The total number of 39 alleles was produced among 40 accessions in the size range of (110-400) bp. Of these, 35 amplification fragments were polymorphic. The level of polymorphism detected was varied from 3 to 6 alleles with an average of 4.22 alleles per locus to distinguish among mango accessions analyzed. Six out of nine microsatellite markers gave the PIC values of greater than (0.5) that can discriminate well the genetic variations among all accessions. Based on their information contents, loci polymorphisms can be divided into three levels: high $(\mathrm{PIC}>0.5)$, medium $(0.5>\mathrm{PIC}>0.25)$, and low (PIC $<0.25$ ) [25]. The polymorphism information content (PIC) value per SSR locus was in the range from 0.368 (MiSHRS 39) to 0.691 (MN36). The highest PIC value of (0.691) is possessed by the MN36 followed by MN24 and MN19 carrying the same PIC values of (0.596), after that MN16 having the PIC value of (0.583), MN89 (0.517), LMMA8 (0.501) and MN84 (0.492). MiSHRS39 (0.368) and MN85 (0.389) held the least PIC value. The average PIC and UHe value detected by 9 microsatellite markers were $(0.526)$ and $(0.561)$ respectively. Many researchers have been widely studied the genetic diversity of mango in both inter and intra-varieties by SSR markers. In the report of Begum et al (2012)[1], it was found that 11 choicest juicy mango cultivars in Andhra Pradesh were analyzed by 10 highly polymorphic SSR markers out of which 6 SSR markers were used in this study. Their resultant analysis revealed that the

Table 2: List of 9 SSR markers used in this study with their respective allele size range and annealing temperature $\left({ }^{\circ} \mathrm{C}\right)$

\begin{tabular}{|c|c|c|c|c|}
\hline S.No. & Primers & Primers $\left(5^{\prime}-3^{\prime}\right)$ & Allele size range (bp) & Annealing temp $\left({ }^{\circ} \mathrm{C}\right)$ \\
\hline 1. & LMMA 8 & $\begin{array}{l}\text { F-CATGGAGTTGTTGATACCTAC } \\
\text { R -CAGAGTTAGCCATATAGAGTG }\end{array}$ & $250-350$ & 45 \\
\hline 2. & MN 36 & $\begin{array}{l}\text { F-CCTCAATCTCACTCAACA } \\
\text { R-ACCCCACAATCAAACTAC }\end{array}$ & $220-400$ & 50 \\
\hline 3. & MN 84 & $\begin{array}{l}\text { F-TCTATAAGTGCCCCCTCACG } \\
\text { R-ACTGCCACCGTGGAAAGTAG }\end{array}$ & $220-350$ & 50 \\
\hline 4. & MN 24 & $\begin{array}{l}\text { F-CGATGGACTTCATAAGAAGAG } \\
\text { R-GCTAGCAGAATCACCTTGGTC }\end{array}$ & $150-180$ & 50 \\
\hline 5. & MN 19 & $\begin{array}{l}\text { F-AATTATCCTATCCCTCGTATC } \\
\text { R-AGAAACATGATGTGAACC }\end{array}$ & $120-200$ & 42 \\
\hline 6. & MN16 & $\begin{array}{l}\text { F-GCTTTATCCACATCAATATCC } \\
\text { R-TCCTACAATAACTTGCC }\end{array}$ & $150-200$ & 42 \\
\hline 7. & MiSHRS39 & $\begin{array}{l}\text { F-GAACGAGAAATCGGGAAC } \\
\text { R-GCAGCCATTGAATACAGAG }\end{array}$ & $340-390$ & 45 \\
\hline 8. & $\mathrm{MN}-85$ & $\begin{array}{l}\text { F-GCTTGCTTCCAACTGAGACC } \\
\text { R-GCAAAATGCTCGGAGAAGAC }\end{array}$ & $250-380$ & 58 \\
\hline 9. & $\mathrm{MN}-89$ & $\begin{array}{l}\text { F-CGCCGAGCCTATAACCTCTA } \\
\text { R-ATCATGCCCTAAACGACGAC }\end{array}$ & $110-200$ & 50 \\
\hline
\end{tabular}


Table 3: Polymorphic characteristic of 9 microsatellite markers

\begin{tabular}{lccc}
\hline Primers & No. of alleles & UHe & PIC \\
\hline LM MA 8 & 4 & 0.513 & 0.501 \\
M N36 & 5 & 0.708 & 0.691 \\
M N84 & 6 & 0.505 & 0.492 \\
MN24 & 4 & 0.611 & 0.596 \\
MN19 & 4 & 0.611 & 0.596 \\
MN16 & 4 & 0.598 & 0.583 \\
MiSHRS39 & 3 & 0.579 & 0.368 \\
M N85 & 5 & 0.398 & 0.389 \\
M 89 & 4 & 0.53 & 0.517 \\
Mean & 4.33 & 0.561 & 0.526 \\
\hline
\end{tabular}

$A N=$ No. of alleles, $U \mathrm{He}=$ Unbiased estimate of the expected

heterozygosity, $\mathrm{PIC}=$ Polymorphic information content

number of allele per locus was the same in range from 3 to 6 with an average of 4.4 but it was found that the PIC value of those 11 inters- cultivars was 0.66 in average and higher than that of the present study. 31 accessions of Beneshan mango using 23 out of 109 mango specific SSRs produced lower number of alleles in a range of 2 to 4 allele per locus and lower PIC value in an average of (0.386) [2] than that of the present observations. In comparison with the results of the above studies, it was likely to have more considerable genetic variation among intra-cultivars of Sein Ta Lone mango by using the selected 9 SSR markers. Kumar M et al (2013) [12] used highly polymorphic LMMA and MiSHRS series in which LMMA8 and MiSHRS 39 were also used in this study. The resultant amplified products were varied from 2 to 4 alleles with 2.70 alleles in average which was lower than that of the present study and PIC value was ranging from (0.320 to 0.774$)$ with an average of $(0.529)$ that was the same in line with that of the present study. The previous study [15] conducted on genetic diversity of the same cultivar- Sein Ta Lone, but in different region, 'Kyaukse' using the same SSR markers. It was stated that the allele number ranged in 4 to 7 alleles with an average of (5.33) allele per locus. Although these two studies used the same primers set, there was a slight variation in allele numbers produced by some selected primers. Although there might be a low degree of genetic variation between mango accessions from Sintgaing and Kyaukse, the average PIC value and unbiased expected heterozygosity (UHe) of Sein Ta Lone cutivars within Sintgaing was slightly higher than that of the result of Kyaukse indicating that the level of genetic variation of Sein Ta Lone mango in Sintgaing region seemed to be higher than that in Kyaukse. The survey of local specialists mentioned that the traditional propagation method, grafting, was commonly used in this region by using 'Yin Kwe' as a root stock. Propagation method was one of the most influencing factors for being heterogeneity among Myanmar mangoes and this factor might be influenced within Sein Ta Lone cultivars in these regions. In 2011, Hirano et al [7] studied genetic variability of two mango varieties 'Sein Ta Lone and Yin Kwe' collected from southern parts of Myanmar (Yangon and Bago divisions) in which clear genetic difference was found within each variety. However, lower level of genetic variability among Sein Ta Lone cultivars has been observed when it compared to the present study. One of the possibilities of difference in genetic variation within Sein Ta Lone cultivars might depend on the history of the traditional variety in the cultivated regions. Due to the long cultivation of Sein Ta Lone in central area of Myanmar in many decades and several different propagation methods had been applied, the considerable genetic variation and differentiation might be occurred within genotypes whereas Sein Ta Lone was recently introduced to lower Myanmar causing the limitation of genetic variation level.

\section{Cluster Analysis and Genetic Relationships}

The dendrogram for 40 accessions among three different location sites revealed their genetic relationship (Figure 2). The genetic distance based on the Jaccard's similarity coefficient was ranging from (0.69) to (1.00). All mango accessions were analyzed based on UPGMA cluster analysis and classified into three main clusters (cluster I, II and III). Cluster I included 16 accessions while cluster II consisted of 14 accessions and cluster III comprised of 10 accessions. Cluster I was further divided into two subclusters, IA and IB containing 14 and 2 accessions respectively. Similarly, cluster II was sub-divided into two sub clusters, IIA for 4 accessions and IIB for 10 accessions and cluster III into IIIA and IIIB holding 6 and 4 accessions respectively.

Considering based on UPGMA dendrogram, the 10 mango accessions from 5 orchards in Sunye were only found in main cluster I (IA, IB). Among them, (SR10 and SR18) accessions from Sunye orchard revealed $(100 \%)$ genetic similarity. The accessions ( $\mathrm{SMgl}$ and $\mathrm{SMg} 4$ ) from $\mathrm{U} \mathrm{Mg} \mathrm{Mg}$ orchard emerged as sub cluster IB that was similar at $95 \%$. The remaining aaccessions from 3 orchards: U Aye Ko (SKO4, SKO9), U Phoo Toe (SWl and SW2) and U Chit Shwe (SChl and SCh3) were found together at genetic similarity (GS) coefficient of (0.93). It was interestingly to note that there was a close genetic relationship among the accessions in Sunye because they shared in the same cluster with (0.93 to 1.00$)$ similarity coefficient.

The 10 mango accessions from Paleik were found in two main clusters II (IIA, IIB) and III (IIIB). The accessions (SPi 3, SPi 6) and (SWS 7, SWS 11) collected from Pi TaukYeik and WarSo orchards respectively were clustered as sub cluster IIA with $82 \%$ similarity. The sub cluster IIB comprised of 5 different orchards from both Paleik and Sintgaing. ThazinNwe (STZ14 and STZ 18), U Karka (SKa 2 and SKa6) from Paleik and TaungPawThar (SNMl and SNM3), U Than Oo (STO 4 and STO 8) and Ma Sint (SSi 7 and SSi 12) from Sintgaing shared their genetic similarity at 79\%. The accessions (SKM4 and SKM5) from U Kyaw Myo Tun orchard were found in sub cluster IIIB with (0.89) similarity coefficient. It was found that there was 82- 89\% genetic similarity among different orchards in Paleik.

The 20 accessions from Sintgaing were dispersed throughout the three main clusters I (IA), II (IIB) and III (IIIA,IIIB) as described by Figure 1. Three orchards from Sintgaing: MibaMyittar (SMT1 and SMT5), PawTawMu (SPB3 and SPB4) and PyaeSone (SPS 3 and SPS 4) were clustered together in sub cluster IA at (0.86) similarity coefficient. The mango accessions from three different orchards: Jodu (SJT2 and SJT5), U Shwe Tint (SST2 and SST3), U Bo Than (SBOl and SBO2) orchards were found as sub cluster IIIA 


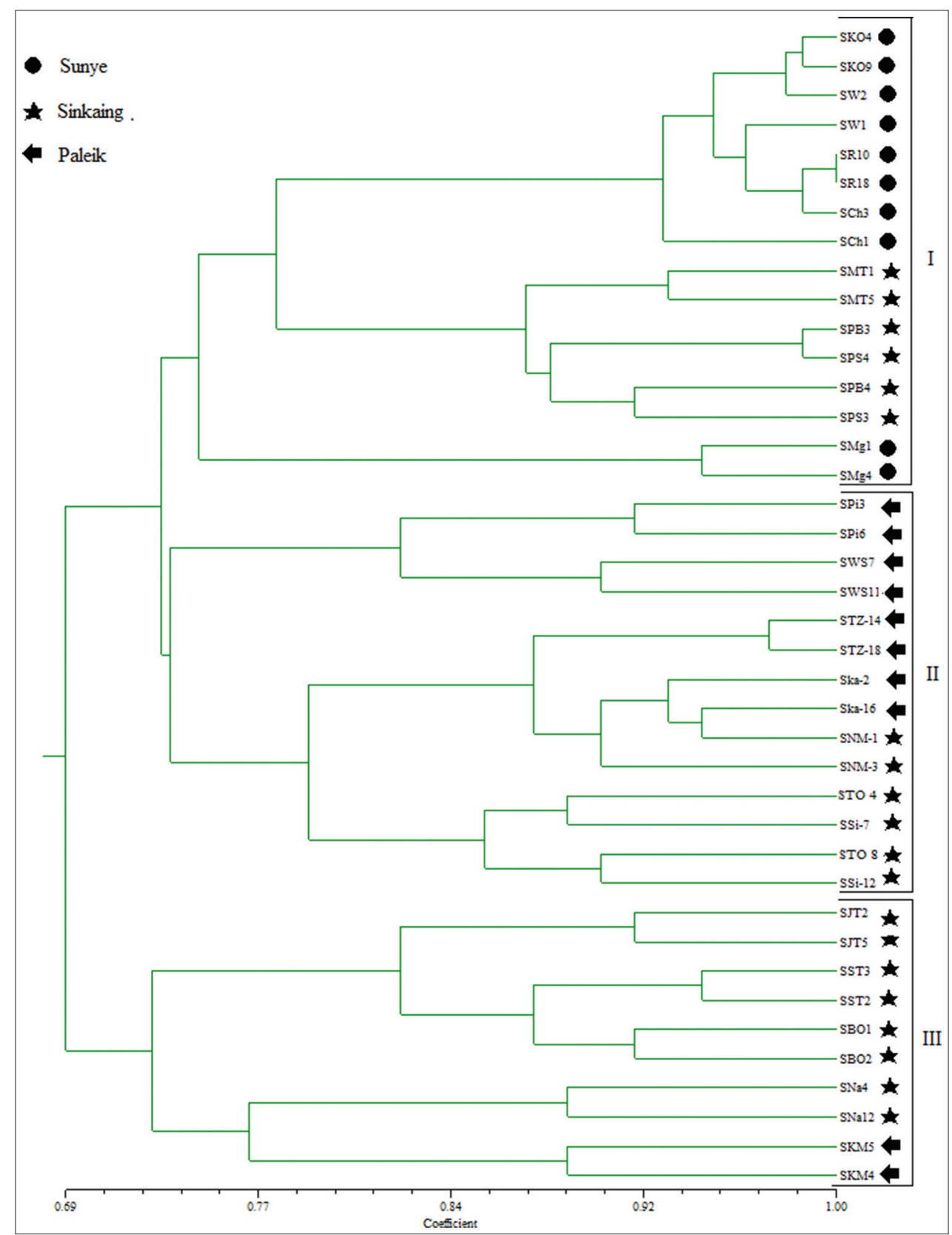

Figure 2: UPGMA dendrogram based on 9 SSR markers for 21'Sein Ta Lone' accessions from Sintgaing Township coded with their respective orchard's owner name: SKO $=U$ Aye Ko, SW = U Phoo Toe, SR = Sunye, SCh $=$ U Chit Swe, SMT = MibaMyitter, SPB $=$ PawTawMu, SPS $=$ PyaeSone, SMg = U Maung Maung, SPi $=$ PiTaukYeik, SWS $=$ WarSo, STZ $=$ Thazin Nwe, SKa $=$ U KarKa, SNM $=$ TaungPawThar, STO $=U$ Than Oo, SSi $=$ Ma Sint, SJT $=$ Jodu, SST $=$ U Swe Tint, SBO = U Bo Than, SNa = Nay Chi Oo, SKM = U Kyaw Myo Tun

at (0.825) similarity coefficient. NayChiOo orchard ( $\mathrm{SNa} 4$ and SNa12) from Sintgaing were similar at (0.89) coefficient and formed grouping together with U Kyaw Myo Tun orchard from Paleik (SKM4 and SKM5) and shared their similarity coefficient of $(0.765)$. It was observed that the different orchards in Sintgaing owed the GS coefficient of (0.825 to $0.89)$. The accessions under the same cluster or sub cluster shared the same genetic background. It was found that the mango accessions from different orchards in Sunye and Sintgaing were similar at (0.78) GS coefficient while the accessions in Sintgaing and Paleik can be seen mixing together in sub cluster IIB and IIIB and similar at genetic similarity coefficient of (0.765 to 0.79 ). According to UPGMA cluster analysis, Jaccard's similarity coefficient revealed moderate level of intracultivar polymorphism $(31 \%)$ which is the probable reason for heterogeneity. This was comparable to the report of Paing Htway HT et al. (2018) [15] in which genetic similarity coefficient of (0.68-0.96) was found among 60 accessions in Kyaukse. Although intracultivar heterogeneity was found throughout Sintgaing Township, there was close genetic relationship within individual orchards. This might be dependent on the private selection of local orchardist who has propagated their own desirable genotypes through conventional breeding. 


\section{CONCLUSIONS}

As a regional polymorphism assessment study, 40 accessions of Sein Ta Lone mango in Sintgaing Township were analyzed for their genetic diversity and relationship by 9 microsatellite markers. The genetic relationship between accessions was in a range of 0.69 to 1.00 and dissimilarity coefficient (0.31) of intracultivar heterogeneity was observed in 40 accessions of Sein Ta Lone mango cultivated throughout Sintgaing Township. This investigation revealed that there might be variation in heterogeneity within intracultivar of Sein Ta Lone in the collected area but it was found that there was close genetic relationship within intracultivars cultivated in individual orchards. This study could be valuable resource for further improvement of this cultivar and further researches are recommended with large numbers of samples and primers set to get more precise information.

\section{ACKNOWLEDGEMENT}

We appreciate the Biotechnology Research Department (BRD), Department of Research and Innovation (DRI), Ministry of Education, Kyaukse, Myanmar for their financial support and laboratory facilities. Heartfelt thanks go to Dr. San San Yu for her kind encouragement and supervision. Deep thanks to Dr. Khin Maung Saing for his invaluable suggestions and comments to succeed this study. Finally, we all would like to express our special thanks to Mango specialist U Myint Htwe and Mango Orchardists in Sintgaing Township for their helpful cooperation.

\section{REFERENCES}

1. Begum H, Reddy MT, Surapaneni M, Reddy BP, Arcahk S, Nagaraju J, Siddiq EA. Molecular analysis for genetic distinctiveness and relationships of indigenous landraces with popular cultivars of mango (Mangifera indica L.) in Andhra Pradesh, India. Asian-Australasian Journal of Bioscience and Biotechnology. 2012; 6: 24-37.

2. Begum H, Reddy MT, Malathi S, Reddy BP, Narshimulu G, Nagaraju J, Siddiq EA. Morphological and microsatellite analysis of intravarietal heterogeneity in 'Beneshan' mango (Mangifera indica L.). Int J Agric Food Res. 2014; 3: 16-33.

3. Dillon NL, Baly ISE, Wright CL, Hucks, L, Ines DJ, Dietzgen RG. Genetic diversity of the Australian national mango genebank, Scientia Horticulturae. 2013; 150: 213-226.

4. Eiadthong W, Yonemori K, Kansaki S, Sugiura A, Utsunomiya N, Subhadrabandhu S. Amplified fragments length polymorphism analysis for studying genetic relationships among Mangifera species in Thailand. Journal of the American Society for Horticultural Science. 2000: 125:160-164.

5. Gupta PK and Varshney RK. Cereal genomics: an overview. In Cereal genomics, edited by Gupta PK and Varshney, RK, 639. Dordrecht, The Netherlands: Kluwer Academic Press; 2004.

6. Hirano R, Htun Oo T, Watanabe K. Myanmar mango landraces reveal genetic uniqueness over common cultivars from Florida, India, and Southeast Asia. Genome.2010; 53:321-330.

7. Hirano R, Ishii H, Htun Oo T, Gilani S, Kikuchi A, Watanabe K.
Propagation management methods have altered the genetic variability of two traditional mango varieties in Myanmar as revealed by SSR. Plant Genetic Resources. 2011; 9(3):404-410. doi:10.1017/ S14792 62111000049

8. Karihaloo JL, Dwivedi YK, Archak S and Baldev Gaikwad A. Analysis of Indian mango cultivars using RAPD markers. The Journal of Horticultural Science and Biotechnology.2003; 78: 285-289.

9. Kashkush K, Jinggui F, Tomer E, Hillel J, Lavi U. Cultivar identification and genetic map of mango (Mangifera indica). Euphytica. 2001; 122: 129-136.

10. Kit YS and Chandran S. A simple, rapid and efficient method of isolating DNA from Chokanan mango (Mangifera indica L.) African Journal of Biotechnology. 2010; 9(36):5805-5808.

11. Kumar H, Narayanswamy P, Prasad T, Mukunda GK, Sondur S. Estimation of genetic diversity of commercial mango cultivars using RAPD markers. Journal of Horticultural Science and Biotechnology. 2001; 76: 529-533.

12. Kumar $M$, Ponnuswam $V$, Nagarajan $P$, Jeyakumar $P$ and Senthil $N$. Molecular characterization of ten mango cultivars using simple sequences repeat (SSR) markers. African Journal of Biotechnology. 2013; 12(47):6568-6573.

13. Myat K. Export conditions of Myanmar mango: hindrances and opportunities in the supply chain. Master Thesis Dissertation, Faculty of Agriculture, University of Bologna, Italy; 2012.

14. Nei M, Roy Choudhury AK. Sampling variances of heterozygosity and genetic distance. Genetics. 1974; 76: 379-390.

15. Paing Htway HT, Yee Soe AN, Myint MM, Yi KP, Nyein Chan NN, et al. Genetic Diversity and Genetic Uniqueness of Indigenous Myanmar Mango (Sein Ta Lone) Cultivar in Kyaukse District. Int J Plant Biol Res. 2018; 6(3): 1089

16. Pandey SN. Mango cultivars, in Srivastav, R.P.P. (Ed.) Mango Cultivation, International Book Distributing Company, Lucknow, India. 1998; 39-99.

17. Pandit SS, Mitra S, Giri AP, Pujari KH, Patil BP, Jambhale ND and Gupta VS. Genetic diversity analysis of mango cultivars using inter simple sequence repeat markers, Current Science. 2007; 93:1135-1141.

18. Popenoe W. Manual of Tropical and Sub topical Fruits, MacMillan, New York, USA; 1920.

19. Ravishankar KV, Mani, BHR, Anand L and Dinesh MR. Development of new microsatellite markers from mango (Mangifera indica) and cross-species amplification. Americian Journal of Botany. 2011; 98:e96-e99.

20. Rohlf FJ. NTSYS-pc: Numerical taxonomy and multivariate analysis system, version 2.1 Exeter Software, Setauket, New York, USA; 2000

21. Schnell RJ, Brown SJ, Olano CT, Meerow AW, Campbell RJ and Kuhn DN. Mango genetic diversity analysis and pedigree inferences for Florida cultivars using microsatellite markers. Journal of the American Society for Horticultural Science. 2006; 13: 214-224.

22. Singh AK, Devanshi Sharma P, Singh R, Singh B, Koundal KR, Singh NK. Assessment of Genetic Diversity in Ziziphus mauritiana using Inter-Simple Sequence Repeat Markers, Journal of Plant Biochemistry and Biotechnology. 2007;16:35-40.

23. Singh S, Bhat KV. Molecular Characterization and analysis of geographical differentiation of Indian Mango (Mangifera indica L) germplasm, Acta Horticulturae (ISHS). 2008; 839: 599-606.

24. Soe TT. Studies on Improved Methods of Postharvest Storage of Mango Fruits. PhD Thesis Dissertation Graduate School of Agriculture Tokyo University of Agriculture Japan; 2006.

25. Vaiman D, Mercier D, Moazami-Goudarzi K, Eggen A, Ciampolini R, Lépingle A, Velmala R, Kaukinen J, Varvio S, Martin P. A set of 99 cattle microsatellites: Characterization, synteny mapping, and polymorphism. Mamm. Genome. 1994; 5: 288-297.

26. Vavilov NI. Centres of origin of cultivated plants, bulletin of applied botany of genetics and plant-breeding. 1926; 16: 1-24. 